\title{
A uniform approach to complexes arising from forests
}

\author{
Mario Marietti \\ Sapienza Università di Roma \\ Piazzale A. Moro 5, 00185 Roma, Italy \\ marietti@mat.uniroma1.it
}

\author{
Damiano Testa \\ Jacobs University Bremen \\ Campus Ring 1, 28759 Bremen, Germany \\ d.testa@jacobs-university.de
}

Submitted: Apr 11, 2008; Accepted: Jul 28, 2008; Published: Aug 4, 2008

Mathematics Subject Classification: 57Q05, 05C05

\begin{abstract}
In this paper we present a unifying approach to study the homotopy type of several complexes arising from forests. We show that this method applies uniformly to many complexes that have been extensively studied in the recent years.
\end{abstract}

\section{Introduction}

In the recent years several complexes arising from forests have been studied by different authors with different techniques (see $[\mathrm{EH}],[\mathrm{E}],[\mathrm{K} 1],[\mathrm{K} 2],[\mathrm{MT}],[\mathrm{W}]$ ). The interest in these problems is motivated by applications in different contexts, such as graph theory and statistical mechanics ([BK], [BLN], $[\mathrm{J}]$ ). We introduce a unifying approach to study the homotopy type of many of these complexes. With our technique we obtain simple proofs of results that were already known as well as new results. These complexes are homotopic to wedges of spheres of (possibly) different dimensions and include, for instance, the complexes of directed trees, the independence complexes, the dominance complexes, the matching complexes, the interval order complexes. In all cases our method provides a recursive procedure to compute the exact homotopy type of the simplicial complex. The dimensions of the spheres arising with these constructions are often strictly related to well-known graph theoretical invariants of the underlying forest such as the domination number, the independent domination number, the vertex covering number and the matching number. Thus we give a topological interpretation to these classical combinatorial invariants.

The paper is organized as follows. Section 2 is devoted to notation and background. In Section 3 we introduce the two basic concepts of this paper: the simplicial complex properties of being a grape (topological or combinatorial) and the strictly related notion of domination between vertices of a simplicial complex. In Section 4 we discuss several applications of these notions: we treat the case of the complex of oriented forests, the independence complex, the dominance complex, the matching complex, edge covering complex, edge dominance complex, and the interval order complex. 


\section{Notation}

Let $G=(V, E)$ be a graph (finite undirected graph with no loops or multiple edges). For all $S \subset V$, let $N[S]:=\{w \in V \mid \exists s \in S,\{s, w\} \in E\} \cup S$ be the closed neighborhood of $S$; when $S=\{v\}$, then we let $N[v]=N[\{v\}]$. If $S \subset V$, then $G \backslash S$ is the graph obtained by removing from $G$ the vertices in $S$ and all the edges having a vertex in $S$ as an endpoint. Similarly, if $S \subset E$, then $G \backslash S$ is the graph obtained by removing from $G$ the edges in $S$. If $S$ is the singleton containing the vertex $v$ or the edge $e$, we also write respectively $G \backslash v$ or $G \backslash e$ for $G \backslash S$. A vertex $v \in V$ is a leaf if it belongs to exactly one edge. A set $D \subset V$ is called dominating if $N[D]=V$. A set $D \subset V$ is called independent if no two vertices in $D$ are adjacent, i.e. $\left\{v, v^{\prime}\right\} \notin E$ for all $v, v^{\prime} \in D$. A vertex cover of $G$ is a subset $C \subset V$ such that every edge of $G$ contains a vertex of $C$. An edge cover of $G$ is a subset $S \subset E$ such that the union of all the endpoints of the edges in $S$ is $V$. A matching of $G$ is a subset $M \subset E$ of pairwise disjoint edges.

We consider the following classical invariants of a graph $G$ which have been extensively studied by graph theorists (see, for instance, [AL], [ALH], [BC], [ET], [HHS], [HY]); we let

- $\gamma(G):=\min \{|D|, D$ is a dominating set of $G\}$ be the domination number of $G$;

- $i(G):=\min \{|D|, \quad D$ is an independent dominating set of $G\}$ be the independent domination number of $G$;

- $\alpha_{0}(G):=\min \{|C|, C$ is a vertex cover of $G\}$ be the vertex covering number of $G$;

- $\beta_{1}(G):=\max \{|M|, M$ is a matching of $G\}$ be the matching number of $G$.

Recall the following well-known result of König (cf [D], Theorem 2.1.1).

Theorem 2.1 (König). Let $G$ be a bipartite graph. Then $\alpha_{0}(G)=\beta_{1}(G)$.

We refer the reader to $[\mathrm{Bo}]$ or $[\mathrm{D}]$ for all undefined notation on graph theory.

Let $X$ be a finite set.

Definition 2.2. A simplicial complex $\Delta$ on $X$ is a set of subsets of $X$, called faces, such that, if $\sigma \in \Delta$ and $\sigma^{\prime} \subset \sigma$, then $\sigma^{\prime} \in \Delta$. The faces of cardinality one are called vertices.

We do not require that $x \in \Delta$ for all $x \in X$.

Every simplicial complex $\Delta$ on $X$ different from $\{\emptyset\}$ has a standard geometric realization. Let $W$ be the real vector space having $X$ as basis. The realization of $\Delta$ is the union of the convex hulls of the sets $\sigma$, for each face $\sigma \in \Delta$. Whenever we mention a topological property of $\Delta$, we implicitly refer to the geometric realization of $\Delta$ with the topology induced from the Euclidean topology of $W$.

As examples, we mention the $(n-1)$-dimensional simplex $(n \geq 1)$ corresponding to the set of all subsets of $X=\left\{x_{1}, \ldots, x_{n}\right\}$, its boundary (homeomorphic to the $(n-2)$-dimensional sphere) corresponding to all the subsets different from $X$, and the boundary of the $n$-dimensional cross-polytope, that is the dual of the $n$-dimensional 
cube. Note that the cube, its boundary and the cross-polytope are not simplicial complexes. We note that the simplicial complexes $\{\emptyset\}$ and $\emptyset$ are different: we call $\{\emptyset\}$ the $(-1)$-dimensional sphere, and $\emptyset$ the $(-1)$-dimensional simplex, or the empty simplex. The empty simplex $\emptyset$ is contractible by convention.

Let $\sigma \subset X$ and define simplicial complexes

$$
\begin{aligned}
(\Delta: \sigma) & :=\{m \in \Delta \mid \sigma \cap m=\emptyset, m \cup \sigma \in \Delta\}, \\
(\Delta, \sigma) & :=\{m \in \Delta \mid \sigma \not \subset m\} .
\end{aligned}
$$

The simplicial complexes $(\Delta: \sigma)$ and $(\Delta, \sigma)$ are usually called respectively link and face-deletion of $\sigma$. If $\Delta_{1}, \ldots, \Delta_{k}$ are simplicial complexes on $X$, we define

$$
\operatorname{join}\left(\Delta_{1}, \ldots, \Delta_{k}\right):=\left\{\cup_{m_{i} \in \Delta_{i}} m_{i}\right\} .
$$

If $x, y \in X$, let

$$
\begin{aligned}
A_{x}(\Delta) & :=\operatorname{join}(\Delta,\{\emptyset, x\}), \\
\Sigma_{x, y}(\Delta) & :=\operatorname{join}(\Delta,\{\emptyset, x, y\}) ;
\end{aligned}
$$

$A_{x}(\Delta)$ and $\Sigma_{x, y}(\Delta)$ are both simplicial complexes. If $x \neq y$ and no face of $\Delta$ contains either of them, then $A_{x}(\Delta)$ and $\Sigma_{x, y}(\Delta)$ are called respectively the cone on $\Delta$ with apex $x$ and the suspension of $\Delta$. If $x \neq y$ and $x^{\prime} \neq y^{\prime}$ are in $X$ and are not contained in any face of $\Delta$, then the suspensions $\Sigma_{x, y}(\Delta)$ and $\Sigma_{x^{\prime}, y^{\prime}}(\Delta)$ are isomorphic; hence in this case sometimes we drop the subscript from the notation. It is well-known that if $\Delta$ is contractible, then $\Sigma(\Delta)$ is contractible, and that if $\Delta$ is homotopic to a sphere of dimension $k$, then $\Sigma(\Delta)$ is homotopic to a sphere of dimension $k+1$. Note that for all $x \in X$ we have

$$
\Delta=A_{x}(\Delta: x) \cup_{(\Delta: x)}(\Delta, x),
$$

where the subscript of the union is the intersection of the two simplicial complexes.

We recall the notions of collapse and simple-homotopy (see $[\mathrm{C}]$ ). Let $\sigma \supset \tau$ be faces of a simplicial complex $\Delta$ and suppose that $\sigma$ is maximal and $|\tau|=|\sigma|-1$ (i.e. $\tau$ has codimension one in $\sigma$ ). If $\sigma$ is the only face of $\Delta$ properly containing $\tau$, then the removal of $\sigma$ and $\tau$ is called an elementary collapse. If a simplicial complex $\Delta^{\prime}$ is obtained from $\Delta$ by an elementary collapse, we write $\Delta \succ \Delta^{\prime}$. When $\Delta^{\prime}$ is a subcomplex of $\Delta$, we say that $\Delta$ collapses onto $\Delta^{\prime}$ if there is a sequence of elementary collapses leading from $\Delta$ to $\Delta^{\prime}$. A collapse is an instance of deformation retract.

Definition 2.3. Two simplicial complexes $\Delta$ and $\Delta^{\prime}$ are simple-homotopic if they are equivalent under the equivalence relation generated by $\succ$.

It is clear that if $\Delta$ and $\Delta^{\prime}$ are simple-homotopic, then they are also homotopic, and that a cone collapses onto a point. 


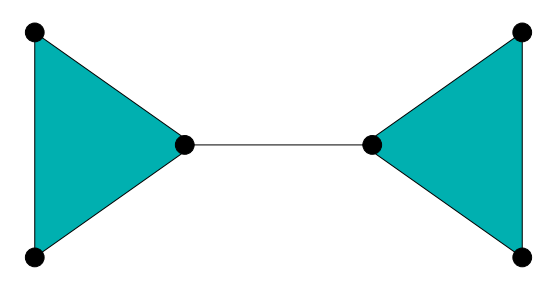

Figure 1: A combinatorial grape

\section{Domination and grapes}

In this section we introduce the notions of grape and domination between vertices of a simplicial complex $\Delta$, and we give some consequences on the topology of $\Delta$.

Let $\Delta^{\prime}$ be a subcomplex of $\Delta ; \Delta^{\prime}$ is contractible in $\Delta$ if the inclusion map $\Delta^{\prime} \hookrightarrow \Delta$ is homotopic to a constant map.

Definition 3.1. A simplicial complex $\Delta$ is a topological grape if

1. there is $a \in X$ such that $(\Delta: a)$ is contractible in $(\Delta, a)$ and both $(\Delta, a)$ and $(\Delta: a)$ are grapes, or

2. $\Delta$ is contractible or $\Delta=\{\emptyset\}$.

Definition 3.2. A simplicial complex $\Delta$ is a combinatorial grape if

1. there is $a \in X$ such that $(\Delta: a)$ is contained in a cone contained in $(\Delta, a)$ and both $(\Delta, a)$ and $(\Delta: a)$ are grapes, or

2. $\Delta$ has at most one vertex.

It follows immediately from the definition that a combinatorial grape is a topological grape. Whenever we say that a simplicial complex is a grape, we shall mean that it is a combinatorial grape.

Note that if $\Delta$ is a cone with apex $b$, then $\Delta$ is a (combinatorial) grape; indeed for any vertex $a \neq b$ we have that both $(\Delta, a)$ and $(\Delta: a)$ are cones with apex $b$, thus $(\Delta: a)$ is contractible in $(\Delta, a)$ and we conclude by induction. It is easy to see that the boundary of the $n$-dimensional simplex is a grape and that the disjoint union of topological or combinatorial grapes is again a grape of the same kind.

There are well-known properties of simplicial complexes that formally resemble the property of being a grape, for instance non-evasiveness, vertex-decomposability, shellability and pure shellability (see $[\mathrm{Bj}, \mathrm{BP}, \mathrm{BW} 1, \mathrm{BW} 2, \mathrm{KSS}]$ ). In general, a grape has none of these properties (see Figure 1 for an example of a grape which is not shellable).

Proposition 3.3. If $\Delta$ is a topological grape, then each connected component of $\Delta$ is either contractible or homotopic to a wedge of spheres. 
Proof. If $\Delta$ is contractible of $\Delta=\{\emptyset\}$, then there is nothing to prove. Otherwise, let $a$ be a vertex such that $(\Delta: a)$ is contractible in $(\Delta, a)$ and both $(\Delta: a)$ and $(\Delta, a)$ are topological grapes. By equation (2.1) and [H, Proposition 0.18] we deduce that $\Delta \simeq(\Delta, a) \vee \Sigma(\Delta: a)$ : indeed attaching the cone with apex $a$ on $(\Delta: a)$ to a contractible space we obtain a space homotopic to the suspension of $(\Delta: a)$. Thus the result follows from the definition of topological grape by induction on the number of vertices of $\Delta$.

In fact we proved that if $a \in X$ and $(\Delta: a)$ is contractible in $(\Delta, a)$, then $\Delta \simeq$ $(\Delta, a) \vee \Sigma(\Delta: a)$. As a consequence, if $\Delta$ is a topological grape, keeping track of the elements $a$ of Definition 3.2, we have a recursive procedure to compute the number of spheres of each dimension in the wedge.

In order to prove that a simplicial complex $\Delta$ is a topological grape we need to find a vertex $a$ such that $(\Delta: a)$ is contractible in $(\Delta, a)$; in the applications it is more natural to prove the stronger statement that there is a cone $C$ such that $(\Delta: a) \subset C \subset(\Delta, a)$ (or equivalently that there is a vertex $b$ such that $\left.A_{b}(\Delta: a) \subset(\Delta, a)\right)$. In the two extreme cases $C=(\Delta, a)$ or $C=(\Delta: a)$, we have $\Delta \simeq \Sigma(\Delta: a)$ or $\Delta \simeq(\Delta, a)$ respectively (in the latter case $\Delta$ collapses onto $(\Delta, a))$. This discussion motivates the following definition.

Definition 3.4. Let $a, b \in X$; $a$ dominates $b$ in $\Delta$ if there is a cone $C$ with apex $b$ such that $(\Delta: a) \subset C \subset(\Delta, a)$.

Definition 3.4 is a generalization of Definition 3.4 of $[\mathrm{MT}]$ which is obtained in the special case in which $C=(\Delta, a)$.

\section{Applications}

In this section we use the concepts introduced in Section 3 to study simplicial complexes associated to forests. We shall see that all these complexes are grapes and are homotopic to wedges of spheres by giving in each case the graph theoretical property corresponding to domination.

\subsection{Oriented forests}

Given a multidigraph $G$, we associate to it a simplicial complex that we call the complex of oriented forests of $G$. This is a generalization of the complex of directed trees introduced in [K1] by D. Kozlov (following a suggestion of R. Stanley). The complex of directed trees is obtained in the special case $G$ is a directed graph. This generalization allows an inductive procedure to work.

A multidigraph $G$ is a pair $(V, E)$, where $V$ and $E$ are finite sets, and such that there are two functions $s_{G}, t_{G}: E \rightarrow V$; we omit the subscript $G$ when it is clear from the context. The elements of $V$ are called vertices, the elements of $E$ are called edges; if $e \in E$, then $s(e)$ is called the source of $e, t(e)$ is called the target of $e$ and $e$ is an edge from $s(e)$ to $t(e)$. We sometimes denote an edge $e$ by $s(e) \rightarrow t(e)$. We usually identify $G=(V, E)$ with $G^{\prime}=\left(V^{\prime}, E^{\prime}\right)$ if there are two bijections $\varphi: V \rightarrow V^{\prime}$ and $\psi: E \rightarrow E^{\prime}$ such 
that $s_{G^{\prime}} \circ \psi=\varphi \circ s_{G}$ and $t_{G^{\prime}} \circ \psi=\varphi \circ t_{G}$. A multidigraph $H=\left(V^{\prime}, E^{\prime}\right)$ is a subgraph of $G$ if $V^{\prime} \subset V, E^{\prime} \subset E$ and $s_{H}, t_{H}$ are the restrictions of the corresponding functions of $G$. A directed graph is a multidigraph such that distinct edges cannot have both same source and same target. We associate to a multidigraph $G=(V, E)$ its underlying undirected graph $G^{u}$ with vertex set $V$ and where $x, y$ are joined by an edge in $G^{u}$ if and only if $x \rightarrow y$ or $y \rightarrow x$ are in $E$.

An oriented cycle of $G$ is a connected subgraph $C$ of $G$ such that each vertex of $C$ is the source of exactly one edge and target of exactly one edge. An oriented forest is a multidigraph $F$ such that $F$ contains no oriented cycles and different edges have distinct targets.

Definition 4.1. The complex of oriented forests of a multidigraph $G=(V, E)$ is the simplicial complex $O F(G)$ whose faces are the subsets of $E$ forming oriented forests.

If $e$ is a loop, i.e. an edge of $G$ with source equal to its target, then $O F(G)=O F(G \backslash$ $\{e\})$. Thus, from now on, we ignore the loops. It follows from the definitions that the complex $O F(G)$ is a cone with apex $y \rightarrow x$ if and only if $y \rightarrow x$ is the unique edge with target $x$ and there are no oriented cycles in $G$ containing $y \rightarrow x$.

The following lemma shows that $O F(G)$ has at most one connected component different from an isolated vertex.

Lemma 4.2. If $G=(V, E)$ is a multidigraph and $a_{1}, a_{2}$ are vertices of $O F(G)$ lying in different connected components $T_{1}$ and $T_{2}$ of $O F(G)$, then at least one of $T_{1}$ and $T_{2}$ consists of the single point $a_{1}$ or $a_{2}$.

Proof. Let $a_{1}=s_{1} \rightarrow t_{1}$ and $a_{2}=s_{2} \rightarrow t_{2}$. Since $\left\{a_{1}, a_{2}\right\}$ is not a face of $O F(G)$, one of the following happens:

1. $t_{1}=s_{2}$ and $t_{2}=s_{1}$;

2. $t_{1}=t_{2}$.

Case (1). If $a=s \rightarrow t$ is an edge of $G$, then necessarily $t \in\left\{t_{1}, t_{2}\right\}$ since otherwise $\left\{a_{1}, a\right\}$ and $\left\{a, a_{2}\right\}$ would be faces of $O F(G)$ and $a_{1}$ and $a_{2}$ could not lie in different connected components. So $E$ consists of $a_{1}, a_{2}$ and of edges with target equal to $t_{1}$ or $t_{2}$. If there are no edges with target $t_{1}$ and source different from $s_{1}=t_{2}$, then $T_{2}$ consists of the single point $a_{2}$. If there are no edges with target $t_{2}$ and source different from $s_{2}=t_{1}$, then $T_{1}$ consists of the single point $a_{1}$. On the other hand, if there are both an edge $b_{1}=s_{1}^{\prime} \rightarrow t_{1}$ and an edge $b_{2}=s_{2}^{\prime} \rightarrow t_{2}$ with $s_{i}^{\prime} \neq s_{i}$ for $i=1,2$, then we have a contradiction since $\left\{a_{1}, b_{2}\right\},\left\{b_{2}, b_{1}\right\},\left\{b_{1}, a_{2}\right\}$ would all be faces, and $a_{1}$ and $a_{2}$ would not lie in different connected components.

Case (2). If $s_{1}=s_{2}$ then, for every edge $b,\left\{a_{1}, b\right\}$ is a face if and only if $\left\{a_{2}, b\right\}$ is. Thus $T_{1}$ and $T_{2}$ consist respectively of the single point $a_{1}$ and the single point $a_{2}$ since $a_{1}$ and $a_{2}$ lie in different connected components. Hence we may assume that $s_{1} \neq s_{2}$.

By the same argument as before, $E$ consists of $a_{1}, a_{2}$, edges with target equal to $t_{1}=t_{2}$, and edges of the type $t_{1} \rightarrow s_{1}$ or $t_{2} \rightarrow s_{2}$. If there are no edges of the type $t_{1} \rightarrow s_{1}$, then 
$T_{2}$ consists of the single point $a_{2}$. If there are no edges of the type $t_{2} \rightarrow s_{2}$, then $T_{1}$ consists of the single point $a_{1}$. On the other hand, if there are both an edge $b_{1}=t_{1} \rightarrow s_{1}$ and an edge $b_{2}=t_{2} \rightarrow s_{2}$, then we have a contradiction since $\left\{a_{1}, b_{2}\right\},\left\{b_{2}, b_{1}\right\},\left\{b_{1}, a_{2}\right\}$ would all be faces, and $a_{1}$ and $a_{2}$ would not lie in different connected components.

For any edge $e \in E$, the simplicial complex $(O F(G), e)$ is the complex of oriented forests of the multidigraph $(V, E \backslash\{e\})$. We denote by $G_{\downarrow e}$ the multidigraph obtained from $G$ by first removing the edges with target $t(e)$, and then identifying the vertex $s(e)$ with the vertex $t(e)$. The reason for introducing this multidigraph is that $(O F(G): e)$ is isomorphic to $O F\left(G_{\downarrow e}\right)$. Indeed no face of $(O F(G): e)$ contains an arrow with target $t(e)$ or becomes an oriented cycle by adding $e$; thus there is a correspondence between the faces of the two complexes. We note that if $G$ is a directed graph, then $G_{\downarrow e}$ could be a multidigraph which is not a directed graph.

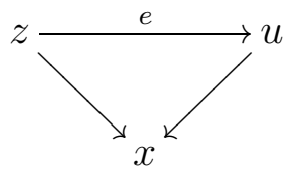

A directed graph $G$

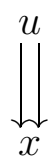

The multidigraph $G_{\downarrow}$

Lemma 4.3. Let $z \rightarrow u$ and $y \rightarrow x$ be distinct vertices of $O F(G)$; then $z \rightarrow u$ dominates $y \rightarrow x$ in $O F(G)$ if and only if one of the following is satisfied:

1. $z=y$ and $u=x$;

2. $u=x$ and there are no oriented cycles containing $y \rightarrow x$;

3. $z=x$, the unique edges with target $x$ other than $y \rightarrow x$ have source $u$, and all oriented cycles containing $y \rightarrow x$ contain also $u$;

4. $x \neq u, z, y \rightarrow x$ is the unique edge with target $x$, and all oriented cycles containing $y \rightarrow x$ contain also $u$.

Proof. It is clear that $e$ dominates $f$ whenever $s(e)=s(f)$ and $t(e)=t(f)$. Thus we assume that $(z, u) \neq(y, x)$.

Let $z \rightarrow u$ dominate $y \rightarrow x$ in $O F(G)$. Suppose that $u=x$. By contradiction, let $C$ be an oriented cycle of $G$ containing $y \rightarrow x$. Then $z \rightarrow u \notin C$ and hence the edges of $C \backslash\{y \rightarrow x\}$ are a face of $(O F(G): z \rightarrow u)$, but the edges of $C$ are not a face of $(O F(G), z \rightarrow u)$ and hence $(O F(G), z \rightarrow u)$ does not contain the cone with apex $y \rightarrow x$ on $(O F(G): z \rightarrow u)$. Suppose now that $u \neq x$. Clearly there can be no edges with target $x$ different from $y \rightarrow x$ or $u \rightarrow z$ in the case $x=z$, since each of these edges forms a face of $(O F(G): z \rightarrow u)$. Let $C$ be an oriented cycle of $G$ containing $y \rightarrow x$. Then the edges of $C \backslash\{y \rightarrow x\}$ are a face of $(O F(G): z \rightarrow u)$ if and only if $C$ does not contain the vertex $u$. Since the edges of $C$ are not a face of $(O F(G), z \rightarrow u)$ we must have that $u$ is a vertex of $C$. 
Conversely, let $\sigma$ be a face of $(O F(G): z \rightarrow u)$. We need to show that $\sigma \cup\{y \rightarrow x\}$ is a face of $(O F(G), z \rightarrow u)$ : equivalently we need to show that it is a face of $O F(G)$, since $\sigma$ does not contain $z \rightarrow u$. We may assume that $y \rightarrow x \notin \sigma$. Suppose first that $u=x$ and there are no oriented cycles containing $y \rightarrow x ; \sigma$ contains no edge with target $x$, since $\sigma \in(O F(G): z \rightarrow u)$ and $\sigma \cup\{y \rightarrow x\}$ is a face of $O F(G)$ since there are no oriented cycles containing $y \rightarrow x$. Suppose now that we are in case (3) or (4). By assumption no edge of $\sigma$ has $x$ as a target; moreover if $C$ is a cycle containing $y \rightarrow x$, then $\sigma$ cannot contain all the edges of $C \backslash\{y \rightarrow x\}$, since one of these edges has target $u$ and so it is not a face of $(O F(G): z \rightarrow u)$.

We call a multidigraph $F$ a multidiforest if its underlying graph $F^{u}$ is a forest. The following result determines the homotopy types of the complexes of oriented forests of multidiforests.

Theorem 4.4. Let $F$ be a multidiforest. Then $O F(F)$ is a grape and it is either contractible or homotopic to a wedge of spheres.

Proof. Proceed by induction on the number of edges of $F$. It suffices to show that $F$ contains two distinct edges $z \rightarrow u$ and $y \rightarrow x$ such that $z \rightarrow u$ dominates $y \rightarrow x$, since both $F \backslash\{z \rightarrow u\}$ and $F_{\downarrow z \rightarrow u}$ are multidiforests.

If $e, f$ are distinct edges with $s(e)=s(f)$ and $t(e)=t(f)$, then $e$ dominates $f$ (and symmetrically $f$ dominates $e$ ) by Lemma 4.3. Thus we may assume that $F$ is a directed graph. Let $y$ be a leaf of $F^{u}$ and let $x$ be the vertex adjacent to $y$. Recall that the complex $O F(F)$ is a cone with apex $a \rightarrow b$ if and only if $a \rightarrow b$ is the unique edge with target $b$ and there are no oriented cycles in $F$ containing $a \rightarrow b$ (i.e. there is no edge with source $b$ and target $a$ ). Since a cone is a grape, we only need to consider two cases:

1. $y \rightarrow x$ and $x \rightarrow y$ are both edges of $F$,

2. $y \rightarrow x$ is an edge of $F, x \rightarrow y$ is not and there is $z \rightarrow x$ with $z \neq y$.

By Lemma 4.3, in case (1) $y \rightarrow x$ dominates $x \rightarrow y$, in case (2) $z \rightarrow x$ dominates $y \rightarrow x$; in both cases we conclude that $O F(F)$ is a grape. The last statement now follows at once by Proposition 3.3 and Lemma 4.2 .

The proof of Theorem 4.4 gives a recursive procedure to compute explicitly the homotopy type of $O F(F)$, i.e. the number of spheres of each dimension. Thus it generalizes [K1, Section 4], where a recursive procedure to compute the homology groups of the complexes of oriented forests of directed trees is given.

Example 4.5. Let $F$ be the directed tree depicted in the following figure. 


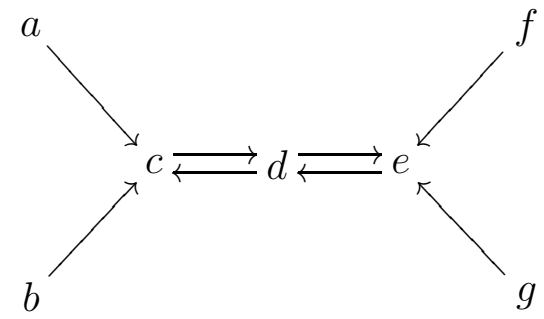

The directed tree $F$

By Lemma 4.3, $d \rightarrow c$ dominates $a \rightarrow c$ and hence $O F(F) \simeq O F\left(F_{1}\right) \vee \Sigma O F\left(F_{2}\right)$, where the directed trees $F_{1}, F_{2}$ are given in the following figure.

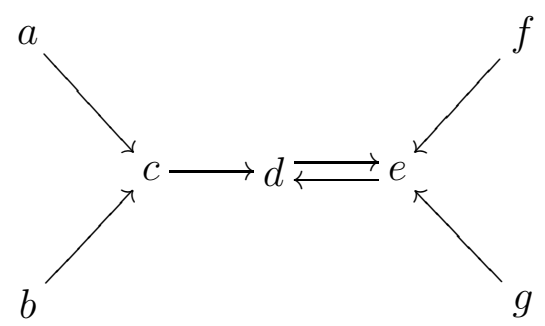

The directed tree $F_{1}$

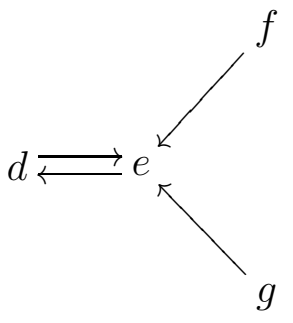

The directed tree $F_{2}$

We consider first $O F\left(F_{2}\right)$. The edge $d \rightarrow e$ dominates $f \rightarrow e$ in $O F\left(F_{2}\right)$; the complex $\left(O F\left(F_{2}\right), d \rightarrow e\right)$ is a cone with apex $e \rightarrow d$, and $\left(O F\left(F_{2}\right): d \rightarrow e\right)=\{\emptyset\}$, since $F_{2 \downarrow d \rightarrow e}$ has no edges different from loops. Hence $O F\left(F_{2}\right) \simeq S^{0}$ (and it is depicted below) and $O F(F) \simeq O F\left(F_{1}\right) \vee S^{1}$.

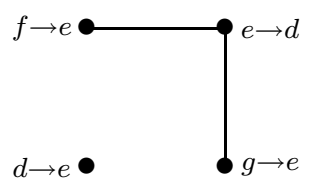

The simplicial complex $O F\left(F_{2}\right)$

Let us now consider $O F\left(F_{1}\right)$. By Lemma 4.3, $a \rightarrow c$ dominates $b \rightarrow c$. Since $\left(O F\left(F_{1}\right), a \rightarrow c\right)$ is a cone with apex $b \rightarrow c$, it follows that $O F\left(F_{1}\right) \simeq \Sigma O F\left(F_{3}\right)$, where $F_{3}$ is depicted in the following figure. 


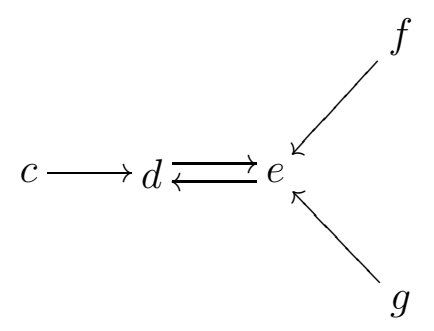

The directed tree $F_{3}$

The edge $e \rightarrow d$ dominates $c \rightarrow d$ in $O F\left(F_{3}\right) ;\left(O F\left(F_{3}\right), e \rightarrow d\right)$ is a cone with apex $c \rightarrow d$, and $\left(O F\left(F_{3}\right): e \rightarrow d\right)$ consists of the two isolated points $f \rightarrow e$ and $g \rightarrow e$. Thus $O F\left(F_{3}\right) \simeq S^{1}$; indeed $O F\left(F_{3}\right)$ is depicted in the following figure.

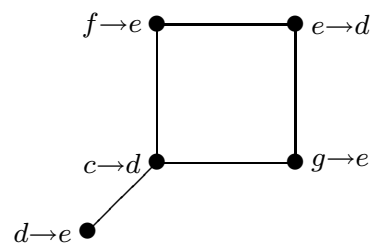

The simplicial complex $O F\left(F_{3}\right)$

Finally the simplicial complex $O F(F)$ is homotopic to $S^{2} \vee S^{1}$.

\subsection{The independence complex}

Let $G=(V, E)$ be a graph. The simplicial complex on $V$ whose faces are the subsets of $V$ containing no adjacent vertices is denoted by $\operatorname{Ind}(G)$ and is called the independence complex of $G$. We have

$$
\begin{aligned}
(\operatorname{Ind}(G), v) & =\operatorname{Ind}(G \backslash\{v\}) \\
(\operatorname{Ind}(G): v) & =\operatorname{Ind}(G \backslash N[v]) .
\end{aligned}
$$

The simplicial complex $\operatorname{Ind}(G)$ is a cone of apex $a$ if and only if $a$ is an isolated vertex of $G$.

Lemma 4.6. Let $a$ and $b$ be vertices of $G$; $a$ dominates $b$ in $\operatorname{Ind}(G)$ if and only if $N[b] \backslash$ $\{b\} \subset N[a]$.

Proof. The faces of $\operatorname{Ind}(G \backslash N[a])$ are the independent sets of vertices of $G \backslash N[a]$. Let $D$ be a face of $\operatorname{Ind}(G \backslash N[a]) ; D \cup\{b\}$ is a face of $\operatorname{Ind}(G \backslash a)$ if and only if $b \in D$ or $b \notin N[D]$. Since this must be true for all faces, $N[b] \backslash\{b\} \cap(V \backslash N[a])=\emptyset$, and the result follows. 
We recall the following result by Engstrom [E].

Lemma 4.7. Let a be a vertex of $G$ having distance two from a leaf $b$. Then $\operatorname{Ind}(G)$ collapses onto $\operatorname{Ind}(G \backslash a)$.

The removal of vertices at distance two from a leaf has also been used by Kozlov for the independence complex of a path and by Wassmer for rooted forests (see [K1] and [W]).

In a forest $F$, a vertex $a$ dominates a vertex $b$ if and only if

1. $b$ is a leaf and $a$ is adjacent to $b$;

2. $b$ is a leaf and $a$ has distance two from $b$;

3. $b$ is isolated.

The third case deals with the trivial case in which $\operatorname{Ind}(F)$ is a cone with apex $b$. Specifying the treatment of the domination to the first case we obtain the analysis of [MT, Section 5]; specifying it to the second case we obtain the analysis of [E] and [W, Section 3.2]. In the first approach what happens is that at each stage the removal of the vertex $a$ and of all its neighbours changes the homotopy type of $\operatorname{Ind}(F)$ by a suspension; thus the relevant informations are the number $r_{1}$ of steps required to reach a graph $F_{1}$ with no edges and the number $i_{1}$ of isolated vertices of $F_{1}$. In the second approach what happens is that at each stage the removal of the vertex $a$ does not change the homotopy type of $\operatorname{Ind}(F)$; thus the relevant informations are the numbers $r_{2}$ and $i_{2}$ of isolated edges and vertices of the graph $F_{2}$ obtained by performing the removal until only isolated vertices or edges are left. The conclusion is that $i_{1} \neq 0$ if and only if $i_{2} \neq 0$ and if and only if $\operatorname{Ind}(F)$ collapses onto a point. If $i_{1}=i_{2}=0$, then $r_{1}=r_{2}=r$ and $\operatorname{Ind}(F)$ collapses onto the boundary of the $r$-dimensional cross-polytope; it can be proved that $r=i(F)=\gamma(F)$, see [MT, Theorem 5.4]. We state explicitly the following result for further reference.

Theorem 4.8. Let $F$ be a forest. Then $\operatorname{Ind}(F)$ is a grape. Moreover, $\operatorname{Ind}(F)$ is either contractible or homotopic to a sphere.

\subsection{The dominance complex}

Let $G=(V, E)$ be graph. The simplicial complex on $V$ whose faces are the complements of the dominating sets is denoted by $\operatorname{Dom}(G)$ and is called the dominance complex of $G$; equivalently the minimal non-faces of $\operatorname{Dom}(G)$ are the minimal elements of $\{N[x] \mid x \in V\}$ . The dominance complex of $G$ is never a cone. Let $a \in V$; we have

$$
(\operatorname{Dom}(G): a)=(\operatorname{Dom}(G \backslash a), N[a] \backslash\{a\}) .
$$

Lemma 4.9. Let $a, b$ be distinct non-isolated vertices of $G$; a dominates $b$ in $\operatorname{Dom}(G)$ if and only if for all $v \in N[b] \backslash N[a]$ there exists $m \in V$ such that $N[m] \backslash\{a\} \subset N[v] \backslash\{b\}$. 
Proof. $(\Rightarrow)$ Let $v \in N[b] \backslash N[a]$ and consider $\sigma:=N[v] \backslash\{b\}$. Since $\sigma \cup\{b\} \notin \Delta$ and $a$ dominates $b$, it follows that $\sigma \notin(\Delta: a)$. Thus there is $m \in V$ such that $N[m] \backslash\{a\} \subset$ $\sigma=N[v] \backslash\{b\}$.

$(\Leftarrow)$ Proceed by contradiction and suppose that $a$ does not dominate $b$; hence there exists $\sigma \in(\Delta: a)$ such that $\sigma \cup\{b\} \notin \Delta$. This means that

1. $\nexists m \in V$ such that $N[m] \subset \sigma \cup\{a\}$,

2. $\exists v \in V$ such that $N[v] \subset \sigma \cup\{b\}$.

If $v$ satisfies $N[v] \subset \sigma \cup\{b\}$, then $N[v] \not \subset \sigma$, since otherwise (1) would not hold. Thus $b \in N[v]$; moreover $a \notin N[v]$, since $N[v] \subset \sigma \cup\{b\}$ and $a \notin \sigma$. Hence $v \in N[b] \backslash N[a]$. By assumption there is $m \in V$ such that $N[m] \backslash\{a\} \subset N[v] \backslash\{b\}$ and hence $N[m] \subset$ $N[v] \cup\{a\} \backslash\{b\} \subset \sigma \cup\{a\}$, contradicting (1).

Lemma 4.10. Let $a, b, c$ be distinct vertices of $G$ and suppose that $N[b]=\{a, b\}$ and $\{a, b, c\} \subset N[a]$. Then $\operatorname{Dom}(G)$ collapses onto $\operatorname{Dom}(G \backslash$ edge $\{a, c\})$.

Proof. The simplicial complex $(\operatorname{Dom}(G), a)$ is a cone with apex $b$. We first show that $\operatorname{Dom}(G)$ collapses onto $(\operatorname{Dom}(G), N[c] \backslash\{a\})$. This is trivial if $N[c] \backslash\{a\}$ is not a face of $\operatorname{Dom}(G)$. Otherwise, let $L=(\operatorname{Dom}(G): N[c] \backslash\{a\}) \subset(\operatorname{Dom}(G), a)$. The simplicial complex $L$ is a cone with apex $b$. Let $\left(\sigma_{1} \supset \tau_{1}\right), \ldots,\left(\sigma_{r} \supset \tau_{r}\right)$ be a sequence of elementary collapses of $L$ to $\emptyset$; adding to $\sigma_{i}$ and $\tau_{i}$ the face $N[c] \backslash\{a\}$ for $1 \leq i \leq r$, we obtain a sequence of elementary collapses of $\operatorname{Dom}(G)$ onto the simplicial complex $(\operatorname{Dom}(G), N[c] \backslash$ $\{a\})$.

We now show that $(\operatorname{Dom}(G), N[c] \backslash\{a\})=\operatorname{Dom}(G \backslash$ edge $\{a, c\})$. The minimal nonfaces of $(\operatorname{Dom}(G), N[c] \backslash\{a\})$ and $\operatorname{Dom}(G \backslash$ edge $\{a, c\})$ are respectively the minimal elements of

$$
\{N[v] \mid v \in V\} \cup\{N[c] \backslash\{a\}\}
$$

and the minimal elements of

$$
\{N[v] \mid v \in V \backslash\{a, c\}\} \cup\{N[c] \backslash\{a\}, N[a] \backslash\{c\}\},
$$

where by $N[v]$ we mean the closed neighborhood of $v$ in the graph $G$. Since $N[b] \subset$ $N[a] \backslash\{c\}$, the minimal elements of the two sets above are the same.

We now consider the dominance complex of a forest $F$. Iterating as long as we can the removal of an edge satisfying the conditions of Lemma 4.10, we obtain a subforest $F^{\prime}$ of $F$ containing only isolated vertices and edges. The forest $F^{\prime}$ depends on the choices of edges; the number $r$ of edges of $F^{\prime}$, though, is independent of the choices by the following result.

Proposition 4.11. Let $F$ be a forest. Then

1. $\operatorname{Dom}(F)$ is a grape; 
2. $\operatorname{Dom}(F)$ collapses onto the boundary of an $r$-dimensional cross-polytope, where $r$ is the number of edges of $F^{\prime}$.

Proof. (1) By Lemma 4.9 the vertex $a$ adjacent to a leaf $b$ dominates $b$, since $N[a] \supset N[b]$. The complex $(\operatorname{Dom}(F), a)$ is a cone with apex $b$, and $(\operatorname{Dom}(F): a)=\operatorname{Dom}(F \backslash a)$. Hence the result follows by induction on the number of vertices.

(2) It follows at once from Lemma 4.10 that $\operatorname{Dom}(F)$ collapses onto $\operatorname{Dom}\left(F^{\prime}\right)$. Since the dominance complex of $F^{\prime}$ is the boundary of the cross-polytope of dimension $r$, where $r$ is the number of edges of $F^{\prime}$, the result follows.

It can be proved that $r=\alpha_{0}(F)=\beta_{1}(F)$ (see [MT, Theorem 6.1]).

\subsection{Matching complex}

Let $G=(V, E)$ be a graph. We define a simplicial complex $M(G)$ on $E$ whose faces are the matchings of $G$, i.e. sets of pairwise disjoint edges. We note that $M(G)$ is the independence complex of the line dual of $G$, i.e. of the graph whose vertices are the edges of $G$ and where $\left\{e_{1}, e_{2}\right\}$ is an edge if $e_{1} \neq e_{2}$ and $e_{1} \cap e_{2} \neq \emptyset$. Note that if $e=\{x, y\} \in E$, then $(M(G), e)=M(G \backslash e)$ and $(M(G): e)=M(G \backslash\{x\} \backslash\{y\})$.

Lemma 4.12. Let $G=(V, E)$ be a graph without cycles of length four. If $e_{1}, e_{2}$ are vertices of $M(G)$ lying in different connected components $T_{1}$ and $T_{2}$ of $M(G)$, then at least one of $T_{1}$ and $T_{2}$ consists of the single point $e_{1}$ or $e_{2}$.

Proof. Since $\left\{e_{1}, e_{2}\right\}$ is not a face of $M(G), e_{1}$ and $e_{2}$ are adjacent. Let $e_{1}=\{u, v\}$ and $e_{2}=\{v, z\}, u \neq z$. Then there is no edge $e$ having empty intersection with $\{u, v, z\}$ since otherwise $\left\{e_{1}, e\right\}$ and $\left\{e, e_{2}\right\}$ would all be faces, and $e_{1}$ and $e_{2}$ would not lie in different connected components.

If there are no edge $f_{1}$ such that $f_{1} \cap\{u, v, z\}=\{z\}$, then $T_{1}$ consists of the single point $e_{1}$. If there are no edge $f_{2}$ such that $f_{2} \cap\{u, v, z\}=\{u\}$, then $T_{2}$ consists of the single point $e_{2}$. On the other hand, if there are both such edges $f_{1}$ and $f_{2}$, then $f_{1}$ and $f_{2}$ are disjoint since $G$ contains no cycles of length four and we have a contradiction because $\left\{e_{1}, f_{1}\right\},\left\{f_{1}, f_{2}\right\},\left\{f_{2}, e_{2}\right\}$ would all be faces, and $e_{1}$ and $e_{2}$ would not lie in different connected components.

If $F$ is a forest, then the line dual of $F$ is not a forest unless $F$ is a disjoint union of paths. Hence Theorem 4.8 does not apply to $M(F)$. Nevertheless, we have the following result.

Theorem 4.13. Let $F=(V, E)$ be a forest. Then $M(F)$ is a grape and it is either contractible or homotopic to a wedge of spheres.

Proof. We proceed by induction on the number of edges of $F$, the base case being obvious. Let $b$ be a leaf and let $a$ be adjacent to $b$. If the edge $\{a, b\}$ is isolated, then $M(F)$ is a cone with apex $\{a, b\}$ and hence it is a grape. Otherwise let $c \neq b$ be adjacent to $a$. By Lemma 4.6, the edge $\{a, c\}$ dominates the edge $\{a, b\}$ in $M(F)$. By induction $M(F)$ is a 
grape since $(M(F),\{a, c\})$ and $(M(F):\{a, c\})$ are matching complexes of forests. The last statement now follows at once by Proposition 3.3 and Lemma 4.12.

Example 4.14. The simplicial complex $M(F)$ may be a wedge of spheres of different dimensions. Let $F$ be the tree depicted in the following figure.

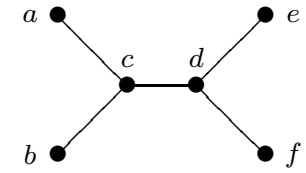

The tree F

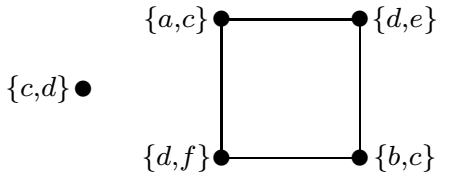

The simplicial complex $M(F)$

The complex $M(F)$ is homeomorphic to $S^{1} \vee S^{0}$.

\subsection{Edge covering complex}

Let $G=(V, E)$ be a graph. We define a simplicial complex $E C(G)$ on $E$ whose faces are the complements of the edge covers of $G$. For all $v \in V$, let $\operatorname{star}(v)=\{e \in E \mid v \in e\}$; thus the minimal non-faces of $E C(G)$ are the minimal elements of $\{\operatorname{star}(v) \mid v \in V\}$. Note that if $G$ has an isolated vertex, then $E C(G)=\emptyset$. Let $e=\{x, y\} \in E$; then $(E C(G): e)=E C(G \backslash e)$ since the minimal non-faces of $(E C(G): e)$ are the minimal elements of

$$
\{\operatorname{star}(v) \mid v \in V, v \neq x, y\} \cup\{\operatorname{star}(x) \backslash\{e\}, \operatorname{star}(y) \backslash\{e\}\} .
$$

The complex $E C(G)$ is a cone with apex $e$ if and only if $x$ and $y$ are both adjacent to leaves.

Theorem 4.15. Let $F$ be a forest. Then $E C(F)$ is a grape. Moreover, $E C(F)$ is either contractible or homotopic to a sphere.

Proof. We may assume that $F$ has no isolated vertices, since $\emptyset$ is a contractible grape. Proceed by induction on the number of edges of $F$. If $F$ is a disjoint union of stars, then $E C(F)=\{\emptyset\}$, the $(-1)$-dimensional sphere. Otherwise, let $x_{1}, \ldots, x_{4}$ be distinct vertices such that $\left\{x_{1}, x_{2}\right\},\left\{x_{2}, x_{3}\right\},\left\{x_{3}, x_{4}\right\}$ are edges and $x_{1}$ is a leaf. If $x_{4}$ is a leaf, then $E C(F)$ is a cone with apex $\left\{x_{2}, x_{3}\right\}$ and we are done. If $x_{4}$ is not a leaf, then $\left\{x_{3}, x_{4}\right\}$ dominates $\left\{x_{2}, x_{3}\right\}$ since $\left(E C(F),\left\{x_{3}, x_{4}\right\}\right)$ is a cone with apex $\left\{x_{2}, x_{3}\right\}$. Hence $\left(E C(F),\left\{x_{3}, x_{4}\right\}\right)$ is a grape, $E C(F)$ is homotopic to the suspension of $E C\left(F \backslash\right.$ edge $\left.\left\{x_{3}, x_{4}\right\}\right)$, and we conclude by the inductive hypothesis.

The following result relates the simplicial complex $E C(F)$ on $E$ to the simplicial complex $\operatorname{Ind}(F)$ on $V$. We let $\kappa(F)$ denote the number of connected components of $F$, namely $\kappa(F)=|V|-|E|$. 
Theorem 4.16. Let $F$ be a forest. Then $E C(F)$ is homotopic to a sphere (resp. contractible) if and only if $\operatorname{Ind}(F)$ is homotopic to a sphere (resp. contractible). Moreover, if $E C(F)$ is not contractible, the dimension of the sphere associated to $E C(F)$ is $i(F)-\kappa(F)-1=\gamma(F)-\kappa(F)-1$.

Proof. We may assume that $F$ has no isolated vertices since in this case $E C(F)=\emptyset$ andInd $(F)$ is a cone, and therefore they are both contractible. Proceed by induction on the number of edges of $F$. If $F$ is a disjoint union of stars, then $E C(F)=\{\emptyset\}$, the $(-1)$-dimensional sphere, and $\operatorname{Ind}(F) \simeq S^{\kappa(F)-1}$ (see [MT, Section 5]). Otherwise, let $x_{1}, \ldots, x_{4} \in V$ be such that $\left\{x_{1}, x_{2}\right\},\left\{x_{2}, x_{3}\right\},\left\{x_{3}, x_{4}\right\}$ are edges and $x_{1}$ is a leaf. If $x_{4}$ is a leaf, then $E C(F)$ is a cone with apex $\left\{x_{2}, x_{3}\right\} ; x_{3}$ dominates $x_{4}$ in $\operatorname{Ind}(F)$ and both $\left(\operatorname{Ind}(F), x_{3}\right)$ and $\left(\operatorname{Ind}(F): x_{3}\right)$ are cones; thus $E C(F)$ and $\operatorname{Ind}(F)$ are both contractible. If $x_{4}$ is not a leaf, then $E C(F)$ is homotopic to $\Sigma\left(E C\left(F^{\prime}\right)\right)$, where $F^{\prime}=$ $F \backslash$ edge $\left\{x_{3}, x_{4}\right\}$, while $\operatorname{Ind}(F)$ is homotopic to $\operatorname{Ind}\left(F^{\prime}\right)$ since both $\operatorname{Ind}(F)$ and $\operatorname{Ind}\left(F^{\prime}\right)$ collapse onto $\operatorname{Ind}\left(F \backslash\left\{x_{3}\right\}\right)$ by Lemma 4.7. By the inductive hypothesis we have that $E C\left(F^{\prime}\right)$ and $\operatorname{Ind}\left(F^{\prime}\right)$ are either both contractible or both homotopic to spheres and thus also $E C(F)$ and $\operatorname{Ind}(F)$ have the same property. Moreover if $E C(F)$ is not contractible, then it is homotopic to a sphere of dimension $\gamma\left(F^{\prime}\right)-\kappa\left(F^{\prime}\right)=\gamma(F)-\kappa(F)-1$. The equalities $i(F)=i\left(F^{\prime}\right)$ and $\gamma(F)=i(F)$, when $E C(F)$ and $\operatorname{Ind}(F)$ are not contractible, follow from [MT, Theorem 5.4].

\subsection{Edge dominance complex}

Let $G=(V, E)$ be a graph. We define a simplicial complex $E D(G)$ on $E$ whose faces are the complements of the dominating sets of the line dual of $G$. For all $e \in E$, let $\operatorname{star}(e)=\{f \in E \mid f \cap e \neq \emptyset\}$; thus the minimal non-faces of $E D(G)$ are the minimal elements of $\{\operatorname{star}(e) \mid e \in E\}$.

Theorem 4.17. Let $F$ be a forest. Then $E D(F)$ is a grape. Moreover $E D(F)$ is homotopic to a sphere of dimension $|E|-\beta_{1}(F)-1=|E|-\alpha_{0}(F)-1$.

Proof. Proceed by induction on the number of edges of $F$. If $F$ consists only of isolated vertices and edges, then $E D(F)=\{\emptyset\}$, the $(-1)$-dimensional sphere, and the result is clear. Let $b$ be a leaf of $F$ and let $\{a, c\}$ be an edge of $F$ such that $a$ is adjacent to $b$ and $c \neq b$. Since $\operatorname{star}(\{a, b\}) \subset \operatorname{star}(\{a, c\})$, we deduce from Lemma 4.9 that $\{a, c\}$ dominates $\{a, b\}$. The complex $(E D(F),\{a, c\})$ is a cone with apex $\{a, b\}$. Since $(E D(F):\{a, c\})=E D(F \backslash$ edge $\{a, c\})$ and $E D(F) \simeq \Sigma(E D(F):\{a, c\})$, we conclude by induction that $E D(F)$ is a grape and that it is homotopic to a sphere. To compute the dimension of the sphere, let $M \subset E$ be a matching of maximum cardinality and $b$ be a leaf adjacent to the vertex $a$. We may assume that the edge $\{a, b\}$ is not isolated. If $\{a, b\} \in M$, then removing an edge $\{a, c\}$ with $c \neq b$ we may conclude by induction. If $\{a, b\} \notin M$, then an edge $\{a, c\} \in M$ for exactly one $c$. The set $M \cup\{a, b\} \backslash\{a, c\}$ is again a matching with same cardinality as $M$, and we may conclude as before. The last equality follows by a similar argument or by Theorem 2.1. 


\subsection{Interval order complex}

Let $X$ be a finite set of closed bounded intervals in $\mathbb{R}$; the interval order complex on $X$ is the simplicial complex $\mathcal{O}(X)$ whose faces are the subsets of $X$ consisting of pairwise disjoint intervals. The simplicial complex $\mathcal{O}(X)$ is nonpure shellable (see $[\mathrm{BM}]$ ). In particular, it follows that $\mathcal{O}(X)$ is contractible or homotopic to a wedge of spheres. We give a short direct computation of the homotopy type of $\mathcal{O}(X)$.

Associated to $X$ there is also a graph $O(X)=(V, E)$, where $V=X$ and $\{I, J\} \in E$ if and only if $I \cap J \neq \emptyset$. Clearly, Ind $(O(X))=\mathcal{O}(X)$.

Lemma 4.18. If $I_{1}, I_{2}$ are vertices of $\mathcal{O}(X)$ lying in different connected components $T_{1}$ and $T_{2}$ of $\mathcal{O}(X)$, then at least one of $T_{1}$ and $T_{2}$ consists of the single point $I_{1}$ or $I_{2}$.

Proof. Since $\left\{I_{1}, I_{2}\right\}$ is not a face of $\mathcal{O}(X), I_{1}$ and $I_{2}$ have non-empty intersection. If every interval in $X$ intersects $I_{1}$, then $I_{1}$ is an isolated vertex of $\mathcal{O}(X)$, and similarly for $I_{2}$. Thus we may assume that $X$ contains intervals $J_{1}$ and $J_{2}$ such that $J_{1} \cap I_{1}=\emptyset$ and $J_{2} \cap I_{2}=\emptyset$. The intersection $J_{1} \cap I_{2}$ is non-empty, since otherwise $\left\{I_{1}, J_{1}\right\},\left\{J_{1}, I_{2}\right\}$ would be faces of $\mathcal{O}(X)$ and $I_{1}$ and $I_{2}$ would not lie in different connected components. Similarly $J_{2} \cap I_{1} \neq \emptyset$. Thus $J_{1} \cap J_{2}=\emptyset$ since they are intervals of $\mathbb{R}$. This is impossible since $\left\{I_{1}, J_{1}\right\},\left\{J_{1}, J_{2}\right\},\left\{J_{2}, I_{2}\right\}$ would all be faces of $\mathcal{O}(X)$.

Theorem 4.8 does not apply to $\operatorname{Ind}(O(X))$, since in general $O(X)$ is not a forest. Nevertheless we have the following result.

Theorem 4.19. The simplicial complex $\mathcal{O}(X)$ is a grape.

Proof. If $X=\emptyset$, then the result is clear. Otherwise let $I=[a, b] \in X$ be an interval such that $b=\min \{y \mid[x, y] \in X\}$. The vertices of $O(X)$ adjacent to $I$ are the intervals of $X$ containing $b$. If no interval in $X \backslash\{I\}$ contains $b$, then $\mathcal{O}(X)$ is a cone with apex $I$ and we are done. Otherwise, let $J \in X$ be an interval containing $b$. By construction we have $N[I] \subset N[J]$ (in the graph $O(X)$ ) and by Lemma 4.6 we deduce that $J$ dominates $I$ in $\mathcal{O}(X)$. Since $(\mathcal{O}(X), J)=\mathcal{O}(X \backslash\{J\})$ and $(\mathcal{O}(X): J)=\mathcal{O}(X \backslash N[J])$, we conclude by induction on the cardinality of $X$.

Theorem 4.19 and Lemma 4.18 imply that $\mathcal{O}(X)$ is either contractible or homotopic to a wedge of spheres.

Example 4.20. The simplicial complex $\mathcal{O}(X)$ may be a wedge of spheres of different dimensions. Let $X=\{[0,2],[0,6],[1,3],[4,7],[5,8]\}$. The graph $O(X)$ and the simplicial complex $\mathcal{O}(X)$ are depicted in the following figure.

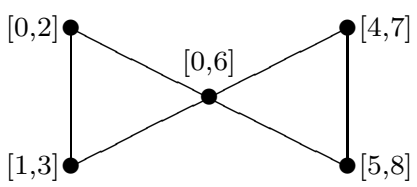

The graph $O(X)$

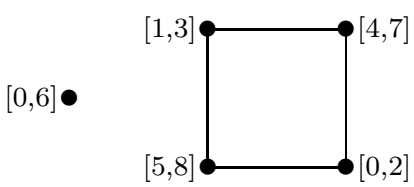

The simplicial complex $\mathcal{O}(X)$ 
The complex $\mathcal{O}(X)$ is homeomorphic to $S^{1} \vee S^{0}$.

\subsection{Summary}

In the following table we summarize the results obtained in this section on the homotopy types of the simplicial complexes associated to a (possibly multidirected) forest $F=(V, E)$ and of the interval order complex. Wedge of spheres means that the spheres have in general different dimensions and the wedge could be empty (i.e. the simplicial complex could be contractible).

\begin{tabular}{|l|l|}
\hline Simplicial Complex & Homotopy type \\
\hline \hline Oriented forests & Wedge of spheres \\
\hline Independence complex & $\begin{array}{l}\text { Contractible or sphere of dimension } \\
i(F)-1=\gamma(F)-1\end{array}$ \\
\hline Dominance complex & $\begin{array}{l}\text { Sphere of dimension } \\
\alpha_{0}(F)-1=\beta_{1}(F)-1\end{array}$ \\
\hline Matching complex & Wedge of spheres \\
\hline Edge covering complex & Contractible or sphere of dimension \\
& $|E|-|V|+i(F)-1=|E|-|V|+\gamma(F)-1$ \\
\hline Edge dominance complex & $\begin{array}{l}\text { Sphere of dimension } \\
|E|-\alpha_{0}(F)-1=|E|-\beta_{1}(F)-1\end{array}$ \\
\hline Interval order complex & Wedge of spheres \\
\hline
\end{tabular}

\section{References}

[AL] Allan, R.B., Laskar, R., On domination and independent domination numbers of a graph, Discrete Mathematics, 23 (1978), 73-76.

[ALH] Allan, R.B., Laskar, R., Hedetniemi S., A note on total domination, Discrete Mathematics, 49 (1984), 7-13.

[BK] Babson, E., Kozlov, D., Proof of the Lovász Conjecture, Ann. of Math. (2) 165 (2007), no. 3, 965-1007.

[BM] Billera, L., Myers, A., Shellability of interval orders, Order 15 (1998/99), no. 2, 113-117.

[BP] Billera, L., Provan, S., A decomposition property for simplicial complexes and its relation to diameters and shellings, Second International Conference on Combinatorial Mathematics (New York, 1978), pp. 82-85, Ann. New York Acad. Sci., 319, New York Acad. Sci., New York, 1979.

[Bj] Björner, A., Topological methods, Handbook of Combinatorics, vol. 2, Elsevier, Amsterdam, 1995, pp. 1819-1872. 
[BW1] Björner, A., Wachs, M., Shellable nonpure complexes and posets. I, Trans. Amer. Math. Soc. 348 (1996), no. 4, 1299-1327.

[BW2] Björner, A., Wachs, M., Shellable nonpure complexes and posets. II, Trans. Amer. Math. Soc. 349 (1997), no. 10, 3945-3975.

[Bo] Bollobás, B., Modern Graph Theory, Graduate Texts in Mathematics, Vol. 184, Springer, 1998.

[BC] Bollobás, B., Cockayne, E.J., Graph-theoretic parameters concerning domination, independence, and irredundance, J. Graph Theory 3 (1979), no. 3, 241-249.

[BLN] Bousquet-Mélou, M., Linusson, S., Nevo, E., On the independence complex of square grids, [math.CO] arXiv:math/0701890v2.

[C] Cohen, M.M., A course in simple-homotopy theory, Graduate Texts in Mathematics, Vol. 10, Springer-Verlag, New York-Berlin, 1973.

[D] Diestel, R., Graph Theory, Graduate Texts in Mathematics, Vol. 173, Springer, 1997.

[EH] Ehrenborg, R., Hetyei, G., The topology of the independence complex, European J. Combin. 27 (2006), no. 6, 906-923.

[E] Engström, A., Complexes of Directed Trees and Independence Complexes, [math.CO] arXiv:math/0508148v1.

[ET] Erdős, P., Tuza, Z., Vertex coverings of the edge set in a connected graph, Graph theory, combinatorics, and algorithms, Vol. 1, 2 (Kalamazoo, MI, 1992), 11791187, Wiley-Intersci. Publ., Wiley, New York, 1995.

[HHS] Haynes, T.W., Hedetniemi, S.T., Slater, P.J., Fundamentals of domination in graphs, Monographs and Textbooks in Pure and Applied Mathematics, 208. Marcel Dekker, Inc., New York, 1998.

[H] Hatcher, A., Algebraic topology, Cambridge University Press, Cambridge, 2002.

[HY] Henning, M.A., Yeo, A., Total domination and matching numbers in claw-free graphs, Electron. J. Combin. 13 (2006), no. 1, Research Paper 59, 28 pp.

[J] Jonsson, J., Hard squares with negative activity and rhombus tilings of the plane, Electron. J. Combin. 13 (2006), no. 1, Research Paper 67, (electronic).

[KSS] Kahn, J., Saks, M., Sturtevant, D., A topological approach to evasiveness, Combinatorica 4 (1984), no. 4, 297-306.

[K1] Kozlov, D., Complexes of directed trees, J. Combin. Theory Ser. A 88 (1999), no. 1, $112-122$.

[K2] Kozlov, D., Directed trees in a string, real polynomials with triple roots, and chain mails, Discrete Comput. Geom. 32 (2004), no. 3, 373-382.

[MT] Marietti, M., Testa, D., Cores of simplicial complexes, Discrete and Computational Geometry, in press (available online 15/05/2008).

[W] Wassmer, A., A Dual Independence Complex, PhD Thesis, Technische Universität Berlin, 2005. 